Document downloaded from:

http://hdl.handle.net/10251/70843

This paper must be cited as:

Kayhan Zrar; Lloret, J. (2015). Frame-based mapping mechanism for energy-efficient MPEG-4 video transmission over IEEE 802.11e networks with better quality of delivery. Journal of Network and Computer Applications. 58:280-286. doi:10.1016/j.jnca.2015.08.005.

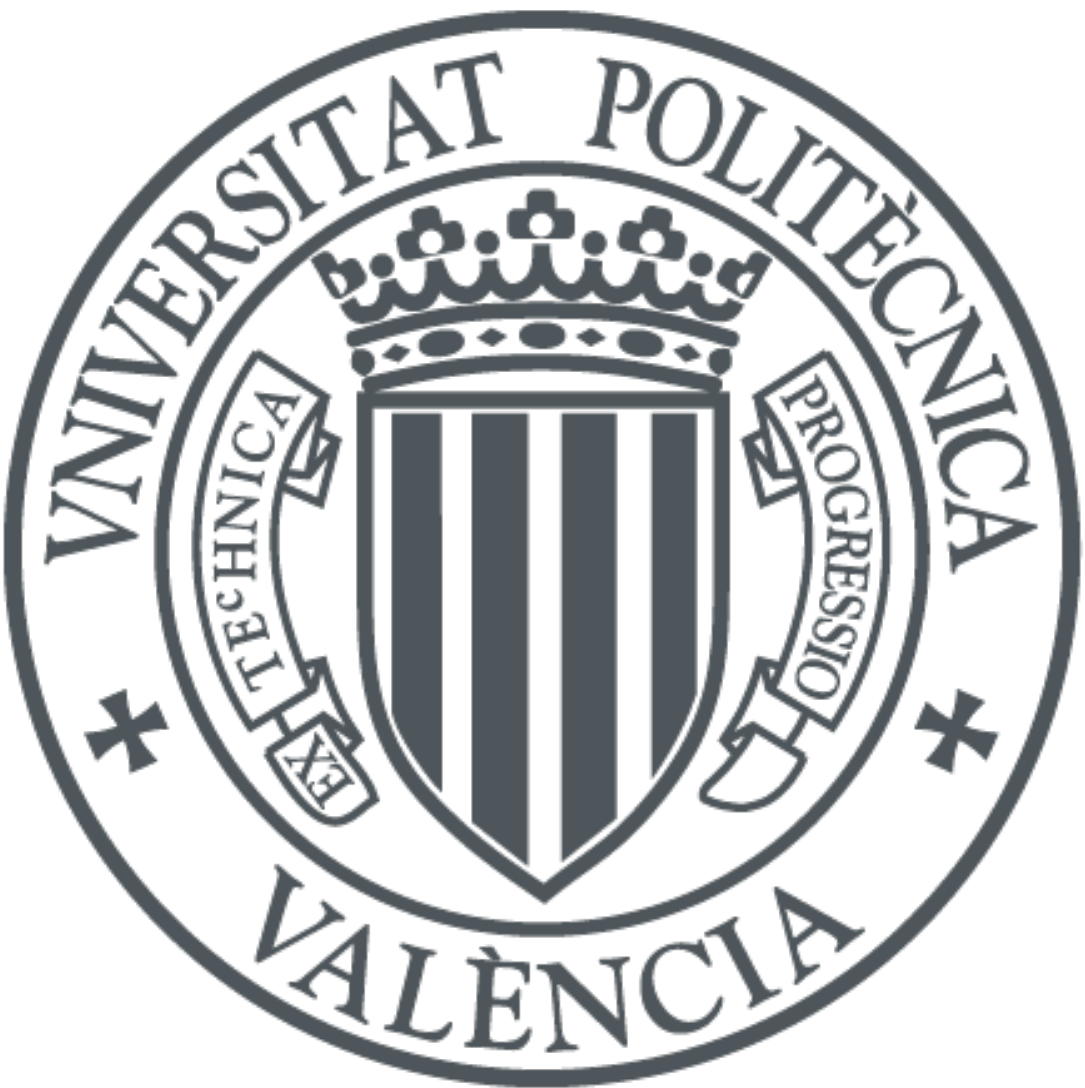

The final publication is available at

http://dx.doi.org/10.1016/j.jnca.2015.08.005

Copyright Elsevier

Additional Information 


\title{
Frame-based Mapping Mechanism for Energy-Efficient MPEG-4 Video Transmission over IEEE 802.11e Networks with Better Quality of Delivery
}

\author{
Chih-Heng Ke \\ Department of Computer Science and Information Engineering, National Quemoy \\ University, Kinmen, Taiwan \\ smallko@gmail.com
}

Chong-Yi Yang, Jiann-Liang Chen

Department of Electrical Engineering, National Taiwan University of Science and

Technology, Taipei, Taiwan

chongyi.yang@gmail.com, lchen@mail.ntust.edu.tw

Kayhan Zrar Ghafoor

Department of Software Engineering, Koya University, Iraq

kayhan@ieee.org

Jaime Lloret

Instituto de Investigación para la Gestión Integrada de zonas Costeras, Universidad Politécnica de Valencia, Spain.

jlloret@dcom.upv.es

Corresponding Author:

Chong-Yi Yang

Department of Electrical Engineering

National Taiwan University of Science and Technology, Taiwan

E-mail: chongyi.yang@gmail.com

\begin{abstract}
Recent developments in hardware, software and communication technologies have resulted in increasing interest in the use of wireless local area networks (WLANs). Mobile devices with embedded WLAN functionality are becoming increasingly popular. Such devices must be designed to support applications that require high quality of service (QoS) and have favorable to maximize battery capacity. The resources of
\end{abstract}


queues in IEEE 802.11e networks may be wasted by the transmission of information that is useless to the receiver. This work develops a frame-based mapping mechanism (FBM) that exploits a different method to process $I / P / B$ video frame packets. FBM refers to the dropping of arriving packets if the preceding packets in the same video frame have been dropped. When fragmented packets of a single frame are allocated to different access categories (AC) queues, out-of-order delivery may occur. Hence, FBM tries to treat all fragmented packets of each video frame equally and allocates them to the same AC queue if possible. The simulation results demonstrate that transmission by the FBM is more efficient than that by other mechanisms, such as EDCA (Enhanced Distributed Channel Access), static mapping and adaptive mapping, suggesting that the energy of a device is not wasted in the transmission of useless video data in WLANs.

\section{Keywords}

Energy efficient, 802.11e, video transmission quality, wireless local area networks.

\section{INTRODUCTION}

WLANs (wireless local area networks) are bandwidth-constrained and less stable than wired LANs, because wireless communication signals can easily suffer from interference. Therefore, given the need to transmit data, multimedia and voice traffic in WLANs, QoS in WLANs must be supported [1][2]. A novel IEEE standard, called IEEE 802.11e, has been introduced to support QoS in WLANs. IEEE 802.11e standard adopts a hybrid coordination function (HCF) protocol, which defines two medium access mechanisms - enhanced distributed channel access (EDCA) and HCF controlled channel access (HCCA). EDCA defines four access categories (ACs) that support QoS by providing differentiated classes of service in the medium access control (MAC) layer; these are voice, video, best effort, and background traffics (AC_VO, AC_VI, AC_BE, and AC_BK, respectively). The transmission of competing queues is prioritized as follows; sound, video, best effort, background. EDCA has four independent queues and sets different channel parameters such as Arbitration Inter Frame Space (AIFS), Contention Window (including $C W_{\min }$ and $C W_{\max }$ ), and Transmission Opportunity Limit (TXOPlimit) for each AC to specify its priority. Multimedia transmissions have strict requirements of delay and bandwidth [3][4]. That is why many researchers are focused on designing and developing new adaptive streaming systems [5]. However, the major impairments of the wireless channel, such as path loss, multipath fading, shadow fading and interference, commonly cause unavoidable burst losses, limited bandwidth and excessive delay. However, no differentiates between different types of video frames in the EDCA, and all packets of video frames of different types are treated equally and mapped into AC_VI. Hence, the loss of some packets of important video frames may reduce the quality of reconstructed data, and even cause video recovery to fail [6]. 
Although IEEE 802.11e is more reliable than the preceding standard, it still refers to the OSI protocol stack, in which layers do not cooperate with each other. To improve the performance of IEEE 802.11e networks, mechanisms have been proposed to adjust the operating parameters, such Contention Window size [7][8], TXOPlimit [9][10] and data transmission rate [11][12]. Some researchers have proposed mechanisms for supporting services that differentiate according to the significance of video frames.

Numerous efficient mechanisms use cross-layers that are based on the features of particular application and the MAC layer [13-15]. Dropping a packet of a video frame in the video server is a more efficient means of avoiding congestion than is dropping it in the MAC layer. This method can be used to transmit video data with the least possible bandwidth and the best video quality [16]. The authors had also proposed scheme for adapting channel variations by using a Real Time Control Protocol (RTCP) report of vehicles within communication range of the video source [17].

This work extends the authors' earlier work $[18,19]$ and develops an efficient means of transmitting MPEG-4 video over IEEE 802.11e networks. Unlike mechanisms in previous works that were developed to reduce the power consumption of stations, the mechanism in this study realizes the efficient transmission of MPEG-4 video over IEEE 802.11e networks, based on the significance of the video frame (frame type) in the application layer. The main difference between the scheme developed in this work and that of $\mathrm{CH}$ Lin et al. [18] is the former tries to treat all fragmented packets of each video frame equally. If the previous packet of a frame is mapped to the AC_VI queue, then the incoming packet will be mapped to the same AC_VI queue. If the previous packet has been dropped, then the incoming packet will also be dropped. Also, the scheme herein is more aggressive than that of $N$ Chilamkurti et al. [20] in protecting important frame packets from being dropped. When the AC_BE or AC_BK queues are becoming congested, the $B$ frame packets are dropped early to reserve more queue space for $I$ or $P$ frame packets. Also, when AC_VI cannot accommodate the $P$ frame packet, this packet will be mapped to the AC_BE or the AC_BK queue, depending on their lengths. This paper also considers the issue of energy-consumption. Although the IEEE 802.11 standard already includes power saving to prolong the operating time and the IEEE 802.11e standard includes QoS support for various types of traffic, further work must be done to improve transmission performance.

This paper is organized as follows. Section 2 discusses the related works. Section 3 describes the proposed frame-based mapping mechanism. Section 4 presents the results of our performance evaluation. Section 5 draws the conclusions and future work.

\section{RELATED WORK}

Several recent works have improved video streaming over the IEEE 802.11e network 
[13][21]. Ksentini et al. proposed a cross-layer framework that prioritized the streaming of H.264 video packets over 802.11e [13]. Static mapping algorithms have been developed for streaming MPEG-4 and H.264/SVC [21]. Video packets are classified according to the priority of the video data. Each class of video packets is assigned a predefined AC. However, as the network condition changes continuously, the static mapping may not work well in a dynamic environment. For example, if the $\mathrm{AC}(2)$ queue is empty and all of the $\mathrm{AC}(1)$ and $\mathrm{AC}(0)$ are full, then the static mapping algorithm will result in the loss of many packets of $P$ and $B$ frames. Restated, static mapping may work well when the video traffic load is heavy and may not be effective when the video traffic load is light but the loads of other types of traffic are heavy. In an enhanced version of adaptive mapping [20] for the H.264 coding scheme, the queue management of $\mathrm{AC}(2)$ is conducted with low and high thresholds for the queue length, and video packets are transmitted to $\mathrm{AC}(1)$ or $\mathrm{AC}(0)$.

Xiao Xiao et al. proposed a dynamic IEEE 802.11e queue scheduling scheme, which adopts a relative queuing delay to allocate a video frame to an appropriate queue. In this work, AC_BE and AC_BK were utilized to transmit video frames [22]. Peng Yu et al. proposed a LADM scheme to improve the quality of transmitted video. In LADM, the enqueued ratio and the dequeued ratio of the video packets are used to quantity the traffic loads. Therefore, the video packets are dynamically mapped to the appropriate access categories according to both the significance of the video frame and the traffic loads [23].

Wen-Ping et al. proposed DVFI+CQM, a novel cross-layer design for streaming high-quality H.264 video over 802.11e ad hoc networks based on the importance of video packets [24]. The DVFI scheme labels the priorities of the video packets in the application layer, and the CQM scheme provides better effective control of congestion among the multiple AC queues in the MAC layer. A simulation shows that DVFI+CQM provides better delivered video quality than 802.11e EDCA in terms of both static mapping and adaptive mapping.

Sunghee et al. proposed a Low Buffer Client First Serve (LBCFS) scheme to improve the quality of Scalable Video Coding (SVC) that is streamed over IEEE 802.11e [25]. For each AC queue, the LBCFS scheme builds a Sub_AC queue that prioritizes the packets based on multimedia stream and the client buffer status. The scheme ensures transmission of the most important frame, which is the $I$ frame.

Xin-Wei et al. proposed an IPB-frame Adaptive Mapping Mechanism (AMM) to improve the quality of video transmitted over IEEE 802.11e WLANs [26]. The mechanism is based on the frame structure of hierarchical coding technology and the traffic load of each AC. The probability that each frame is allocated to the most appropriate AC was dynamically updated according to its importance and the traffic 
load of each AC. Unlike previously proposed mechanisms, AMM dynamically allocated the video frame to a voice AC queue according to its importance and the traffic load of that queue. Although simulation results indicated that AMM outperformed EDCA, the static mapping mechanism and the dynamic mapping mechanism, the benchmark for comparison is different due to the proposed mechanism allocates the frame packets to the AC_VO. That study did not evaluate the influence of video traffic on voice quality (the upper limit on the jitter of voice is 80ms) when AMM allocated the video frame to the voice AC queue.

Most conventional schemes consider only the video frame and the network load and allocate fragmented packets of the same frame to various AC queues, out-of-order delivery may occur. In this work, a frame-based scheme is utilized to improve video transmission quality and is easily adapted to support SVC transmission.

\section{THE PROPOSED FRAME-BASED MAPPING MECHANISM}

The frame-based mapping mechanism $(F B M)$ that is developed herein applies a frame-based algorithm in the sender node of IEEE 802.11e networks, as presented in Figure 1. Packet frame_type $_{n}$ denotes a fragmented packet of a video frame.

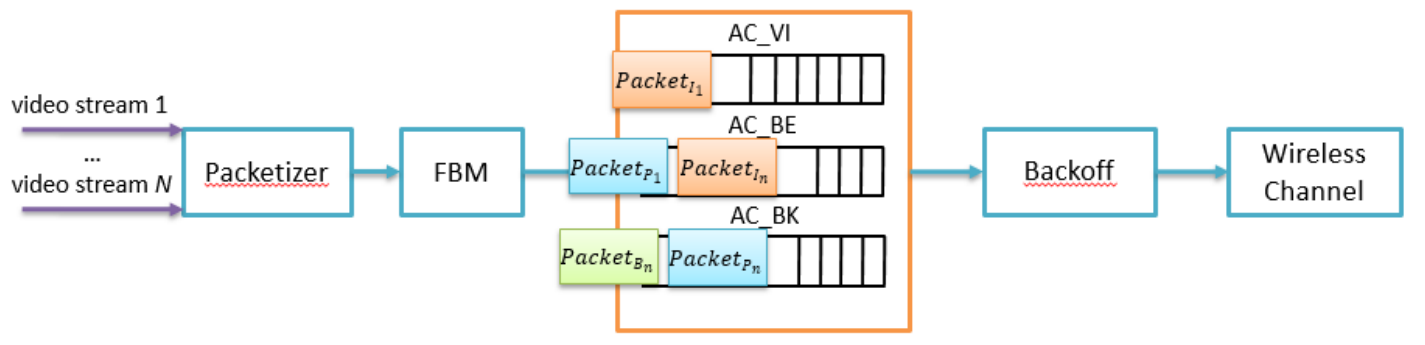

Figure 1. The proposed system framework

The proposed frame-based mapping mechanism (FBM) exploits a different method to process $I / P / B$ video frame packets. First, the packets of frame $I$ are not discarded unless the buffer of the video, the best effort queue, and the background queue are full. Second, FBM adopts an aggressive packet dropping mechanism for frame $B$ to reserve the buffer for the packets of video frames $I$ and $P$ while the network is heavily loaded. Finally, the FBM adopts a frame-based packet mapping mechanism to realize efficient transmission. The FBM refers to the dropping of arriving packets if the preceding packets in the same video frame have been dropped.

FBM dynamically allocates a video packet to an appropriate AC queue, according to the type of video frame, the network traffic load, and the action that had been taken on the previously transmitted packet of the same video frame. Sometimes, the video frame is too large to be transmitted in a single packet. Such a frame has to be fragmented before transmission. Out-of-order delivery may occur when the fragmented packets of the same frame are allocated to different AC queues. Therefore, 
the receiving node must buffer the out-of-order packets of the frame when each fragmented packet of a single frame is treated separately. Hence, all fragmented packets of the same video frame should be treated equally, not separately. The proposed FBM performs the same action as was performed on the preceding packet to the subsequent packet in the same video frame. In this algorithm, the sender node creates a stream table, from which the stream id is determined and records the type of frame and its action. Upon arrival of multiple video frames, the FBM generates a new stream table if a stream id is not found after lookup in the current stream table. To improve the quality of the delivered video, FBM tries to allocate the important video packets to the AC queues with higher priorities in the 802.11e MAC layer. The packet of $I$ frame is allocated to the AC_VI if the AC_VI queue is not full, and the $P$ frame is allocated by referencing Prob. The probability Prob is defined as follows:

$$
\text { Prob }=\frac{\text { qlen }(A C[2])-\text { threshold }}{\left(\text { limit }_{A C[2]}-\text { threshold }\right)}
$$

Equation (1) defines the probability, Prob, that the $P$ frame is mapped to AC(1) or $\mathrm{AC}(0)$ rather than $\mathrm{AC}(2)$ according to the queue length of $\mathrm{AC}(2)$, the threshold and the limit of the queue length. A higher Prob indicates that a packet is being processed in $\mathrm{AC}(1)$ or $\mathrm{AC}(0)$. Table 1 lists the parameters included in equation 1.

Table 1. Parameter notations in our proposed FBM algorithm

\begin{tabular}{|l|l|}
\hline \multicolumn{1}{|c|}{ Parameter } & \multicolumn{1}{c|}{ Description } \\
\hline limit $_{A C(2)}$ & the limit value of queue length of $\mathrm{AC}(2)$ \\
\hline threshold & the threshold of queue length \\
\hline qlen $(A C(2))$ & the queue length of $\mathrm{AC}(2)$ \\
\hline
\end{tabular}

To evaluate the quality of MPEG-4 video that is delivered over IEEE 802.11e networks by the proposed mechanism, two evaluation metrics, PSNR and the proportion of received data that is useless, are used. The decoder may receive useless data from partially received frames or frames that cannot be decoded because they depend on other undecodable frames. The ratio of the useless frames received to the total number of frames received ( $R_{\text {useless }}$ ) is defined as follows:

$$
R_{\text {useless }}=\frac{N_{\text {dec_dir }}-N_{\text {dec }}}{N_{\text {total }}}, 0 \leq R_{\text {useless }} \leq 1
$$


where $N_{\text {total }}$ denotes the total number of video sequence frames; $N_{\text {dec_dir }}$ is the total number of directly decodable frames, and $N_{d e c}$ is the total number of decodable frames, where a frame is considered to be decodable if and only if all of the frames upon which it depends are also decodable27. The resources of a queue in 802.11e networks may be wasted with the transmission of information that becomes useless to the receiver. Therefore, a lower proportion of useless frames received ( $R_{\text {useless }}$ ) corresponds to a more energy-efficient transmission in 802.11e networks.

The energy consumption ( $E_{\text {trans }}$ ) of the WLAN transceiver node is the sum of the energy consumed in various states - transmit $\left(E_{t x}\right)$, receive $\left(E_{r x}\right)$, idle $\left(E_{\text {Idle }}\right)$, sleep $\left(E_{\text {Sleep }}\right)$ and CCA/ED $\left(E_{C C A}\right)$. Equation (3) yields $E_{\text {trans }}$.

$$
\begin{aligned}
E_{\text {trans }}=E_{\text {tx }}+E_{r x}+E_{\text {Idle }}+E_{\text {Sleep }}+ & E_{C C A}=\sum_{i=0}^{N_{t x}} P_{t x} \frac{T_{\text {data }}}{R}+\sum_{i=0}^{M_{r x}} P_{r x} \frac{R_{\text {data }}}{R}+ \\
P_{\text {Idle }} T_{\text {Idle }}+P_{\text {Sleep }} T_{\text {Sleep }}+P_{C C A} T_{C C A} & \text { (3) }
\end{aligned}
$$

where $P_{t x}, P_{r x}, P_{\text {Idle }}, P_{\text {Sleep }}$ and $P_{C C A}$ are the powers in the various states. $T_{\text {Idle }}$, $T_{\text {Sleep }}$ and $T_{C C A}$ denote the intervals for which the transceiver node is in states idle, sleep and CCA respectively. $T_{\text {data }}$ is the length of the $i$-th received packet; $R$ is the data transfer rate, and $N_{t x}$ and $M_{r x}$ are the total numbers of sent and received packets. The energy efficiency $\eta$ is defined as the ratio of the total number of decodable received frames to the energy consumed.

$$
\eta=\frac{N_{d e c}}{E_{\text {trans }}}
$$

Higher $\eta$ indicates that video frames were transmitted more efficiently in the sender node using the mapping mechanism because more video frames were decodable in the receiver node.

Figure 2 presents the flow chart of the proposed FBM algorithm. The FBM exploits different methods for processing $I / P / B$ video frame packets. When a node receives a request to transmit a video frame, the FBM classifies the frame of an arriving video packet. FBM first refers to the action of the preceding frame associated with the same video stream. FBM drops the arriving packets of a frame if the video frame that was transmitted previously in the same stream has already been dropped. A video frame may be regarded as directly undecodable in the receiving node when the packets associated with the video frame that is being received are too few to enable that frame to be decoded. In that case, the FBM directly drops the video packet to reserve the 
buffer for the packets of other video frames.

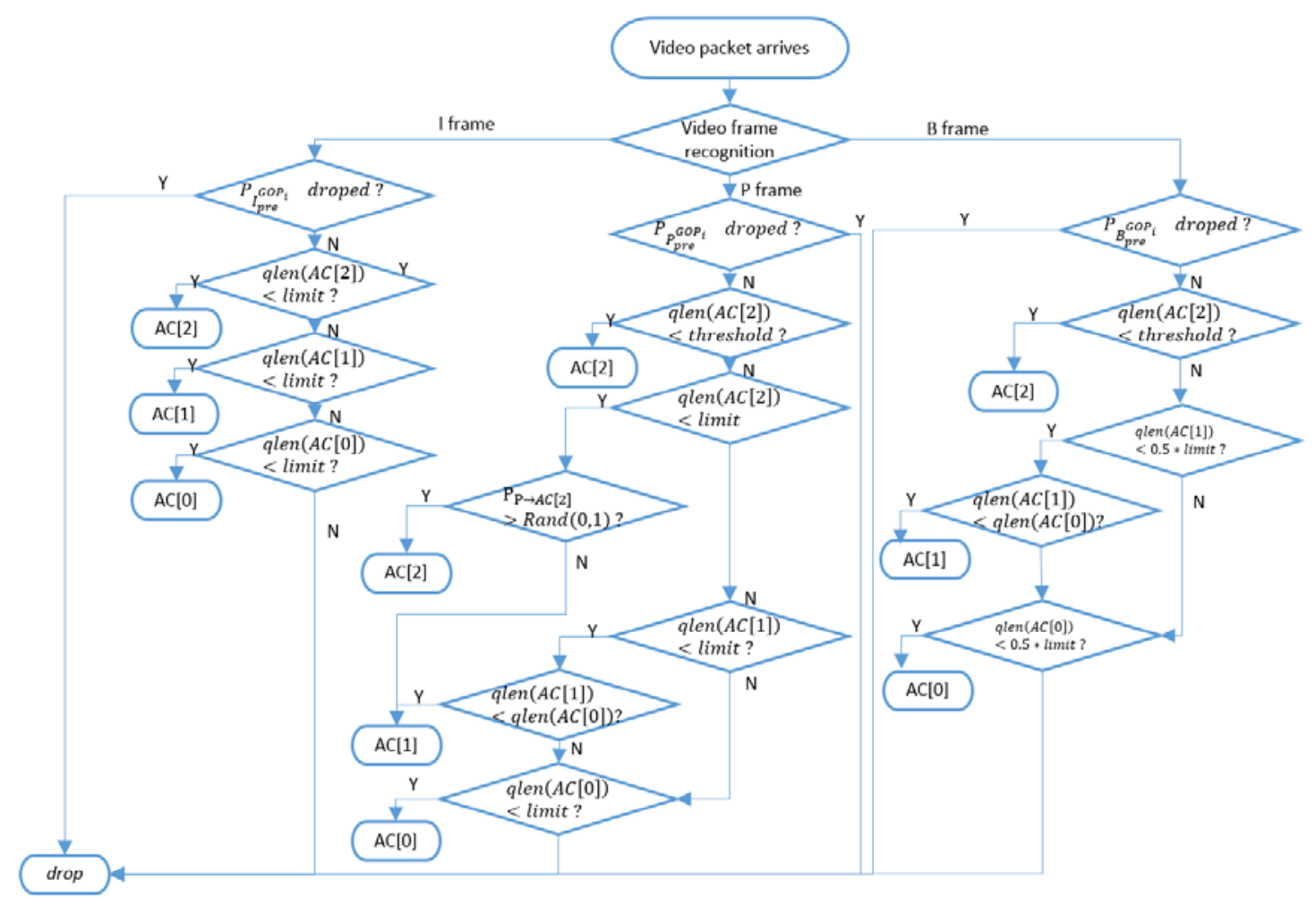

Figure 2. The flow chart of FBM

If packets are associated with a video frame of type $I$, which is importance in both encoding and decoding, then priority will be given to $\mathrm{AC}(2)$ or $\mathrm{AC}(1)$. FBM checks the length of queue $\mathrm{AC}(2)$ and directly maps the packet into $\mathrm{AC}(2)$ when the length of $\mathrm{AC}(2)$ is less than the limiting length of $\mathrm{AC}(2)$. However, when the $\mathrm{AC}(2)$ is full and the qlen $(A C(1))$ is less than limit $_{A C(1)}$, the packet is mapped into $A C(1)$. Subsequently, the video packet will be mapped into $\mathrm{AC}(0)$ when $\mathrm{AC}(0)$ is not full. Otherwise, the video packet will be dropped.

If the packet is associated with a video frame of type $P$, then FBM checks the length of queue $\mathrm{AC}(2)$ and compares it with threshold. If the queue length of $\mathrm{AC}(2)$ is less than threshold, then FBM directly maps the packet into $\mathrm{AC}(2)$. If the queue length of $\mathrm{AC}(2)$ is less than the limit value for $\mathrm{AC}(2)$, then the mapping decision of FBM calculates the value of Prob using equation (1). AC_BE and AC_BK differ in the 802.11e EDCA parameter AIFS. The AIFS value of AC_BE is three and the AIFS of AC_BK is seven. Unlike the difference between the $C W_{\min }$ and $C W_{\max }$ of $\mathrm{AC} \_\mathrm{BE}$ and $\mathrm{AC} \_\mathrm{BK}$, the difference in AIFS is small. Therefore, mapping the packet that has a higher priority into AC_BE may not provide more opportunities to transmit the packet than mapping it into AC_BK. Since the $P$ frame is very important in encoding and decoding, the 
proposed mechanism compares the queue lengths of AC_BE and AC_BK. The FBM directly maps the packet of the $P$ frame to $A C \_B K$ when the queue length of AC_BK is shorter than AC_BE. In contrast, when qlen $(A C(2))$ is closer to threshold, the $P$ frame packet is more likely to be mapped to AC(2).

Finally, if a video packet is associated with a video frame of type $B$, then this packet is mapped into $\mathrm{AC}(2)$ only when the current queue length of $\mathrm{AC}(2)$ is less than the constant threshold. Otherwise, the video packet is mapped into AC_BE or AC_BK when the queue length of AC_BE or AC_BK is less than the half of the constant limit. FBM adopts the early-drop mechanism to drop the packet of the $B$ frame to reserve the buffer for the packets of the I/P frames when the queue lengths of AC_BE and AC_BK currently exceed half of the constant limit. This method of congestion control (follows from OR is derived from) the principle of Random Early Detection (RED) [28] (which is used to predict upcoming congestion), which actively drops packets if required; efficiently prevents congestion, and optimizes queue management.

Lastly, FBM records the action (dropping or queue mapping) of the video packet based on the type of frame (with which it is associated). By exploiting FBM, the mapping strategy and the utilization of network resources depend on the action that is performed on information in the same video stream.

Proposed FBM maps the packets of a video frame to the most appropriate AC and transmit the useful packets of that video frame. FMB is designed to drop the useless packets in the transceiver node to reduce the power consumed by transmission $\left(E_{t x}\right)$ and reserve resources for the useful packets of the video frame.

\section{SIMULATION RESULTS AND DISCUSSION}

\section{A. Simulation Model}

This section evaluates the performance of the proposed frame-based mapping method when it is utilized in various queue management schemes. An NS-2 [29] was conducted to evaluate the performance of the proposed FBM. The default system parameter settings are as follows.

- Video source: YUV CIF (352 × 288), Foreman and Football [30]

- Data rate of wireless link: $11 \mathrm{Mbps}$

- Traffic flow: voice, video, TCP, UDP

- threshold : 40 packets

- Limit (limit queue size of all AC): 50 packets

Two YUV video sources were adopted in the simulation in this study; they were Foreman and Football. Foreman is a short (300 frames) slow-motion video, and Football is an even shorter (260 frames) fast-motion video. Table 2 shows these three 
video sources; indicates the degree of motion, and specifies the numbers of video frames and packets associated with these video sources.

Table 2. The number of video frames and packets of different video sources

\begin{tabular}{|l|c|c|c|c|c|c|c|c|c|c|}
\hline \multirow{2}{*}{ Video } & \multirow{2}{*}{ Format } & \multicolumn{4}{|c|}{ Frame number } & \multicolumn{4}{c|}{ Packets number } & \multirow{2}{*}{ Motion } \\
\cline { 3 - 10 } & & $\boldsymbol{I}$ & $\boldsymbol{P}$ & $\boldsymbol{B}$ & Total & $\boldsymbol{I}$ & $\boldsymbol{P}$ & $\boldsymbol{B}$ & Total & \\
\hline \hline Foreman & CIF & 34 & 67 & 199 & 300 & 268 & 179 & 319 & 766 & \multirow{2}{*}{ Normal } \\
\hline Football & CIF & 34 & 54 & 172 & 260 & 282 & 350 & 725 & 1357 & Fast \\
\hline
\end{tabular}

A scenario is simulated to evaluate video transmission performance. In the scenario, four traffic loads are considered to evaluate the efficacy of $F B M$ under various network loadings. Table 3 presents the different kinds of traffic in each test.

Table 3. number of flows in scenario

\begin{tabular}{|c|c|c|c|c|}
\hline \multirow{2}{*}{ Loads case } & \multicolumn{4}{|c|}{ Number of flows } \\
\cline { 2 - 5 } & Audio (AC(3)) & Video(AC(2)) & TCP (AC(1)) & UDP(AC(0)) \\
\hline \hline Test 1 & 1 & 2 & 1 & 1 \\
\hline Test 2 & 2 & 2 & 2 & 2 \\
\hline Test 3 & 3 & 2 & 3 & 3 \\
\hline Test 4 & 4 & 2 & 4 & 4 \\
\hline
\end{tabular}

\section{B. Simulation results and discussions}

Table 4 shows the average PSNR of the Foreman video, and Table 5 shows the frames and loss of packets of the Foreman video in test 3. The FBM yields the best average PSNR and less frame loss. The EDCA directly allocates packets to AC_VI; adaptive mapping and the FBM allocate packets to AC_VI when the queue of AC_VI is not full or it less than the threshold. Static mapping always allocates $I$ frame to AC_VI, the $P$ frame to AC_BE and the $B$ frame to AC_BK. It does not consider the traffic load of AC queues. The static mapping method drops several packets of the $\mathrm{P}$ and $\mathrm{B}$ frames, resulting in high frame loss, because it allocates the packets to the AC queue with the lowest priority, when the AC queue with the lowest priority has a heavy load.

Table 4. The average PSNR in the scenario Foreman)

\begin{tabular}{|l|c|c|c|c|}
\hline \multirow{2}{*}{ Loads case } & \multicolumn{4}{|c|}{ Average PSNR (db) } \\
\cline { 2 - 5 } & 802.11e EDCA & Static mapping & Adaptive mapping & FBM \\
\hline \hline Test 1 & 33.2 & 31.4 & 33.2 & 33.2 \\
\hline Test 2 & 32.6 & 24.5 & 32.1 & 32.9 \\
\hline
\end{tabular}




\begin{tabular}{|l|l|l|l|l|}
\hline Test 3 & 31.4 & 23.5 & 31.3 & 32.2 \\
\hline Test 4 & 30.3 & 23.4 & 30.2 & 31.5 \\
\hline
\end{tabular}

Table 5. The average PSNR and number of frame and packets lost in test 3 of scenario (Foreman)

\begin{tabular}{|l|c|c|c|c|c|c|c|c|c|}
\hline \multirow{2}{*}{ mechanism } & \multirow{2}{*}{ Average PSNR (db) } & \multicolumn{3}{|c|}{ Frame loss number } & \multicolumn{4}{|c|}{ Packets loss number } \\
\cline { 3 - 10 } & & $\boldsymbol{I}$ & $\boldsymbol{P}$ & $\boldsymbol{B}$ & Total & $\boldsymbol{I}$ & $\boldsymbol{P}$ & $\boldsymbol{B}$ & Total \\
\hline \hline 802.11e EDCA & 30.3 & 6 & 0 & 35 & 40 & 26 & 0 & 60 & 86 \\
\hline Static mapping & 23.4 & 0 & 63 & 134 & 196 & 0 & 154 & 182 & 335 \\
\hline Adaptive mapping & 30.2 & 3 & 3 & 70 & 76 & 5 & 4 & 92 & 100 \\
\hline FBM & 31.5 & 0 & 2 & 54 & 56 & 0 & 5 & 95 & 100 \\
\hline
\end{tabular}

Figure 3 show the average PSNR of the Foreman video in the scenario. Under heavy network loads, FBM still yields a better average PSNR.

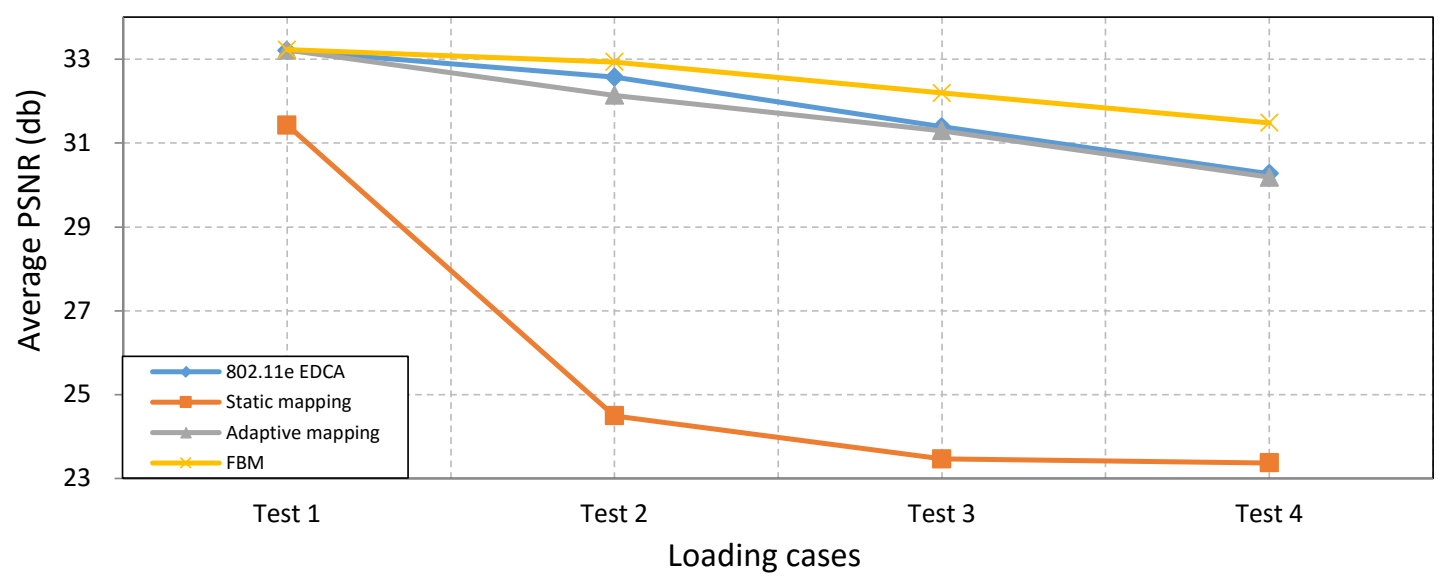

Figure 3. The average PSNR under various loading cases(Foreman)

Figure 4 plots the average useless frame of the video sequence Foreman under various traffic loadings. Under heavy network loads, the FBM still yields the lowest proportion of useless frames in the receiver because FBM does not transmit the packets of the frame in the sender node if the preceding packets in the same video frame have been dropped, and FBM adopts an aggressive packet dropping mechanism for frame $B$ to reserve the buffer for packets of frames $I$ and $P$ when the network is heavily loaded. 


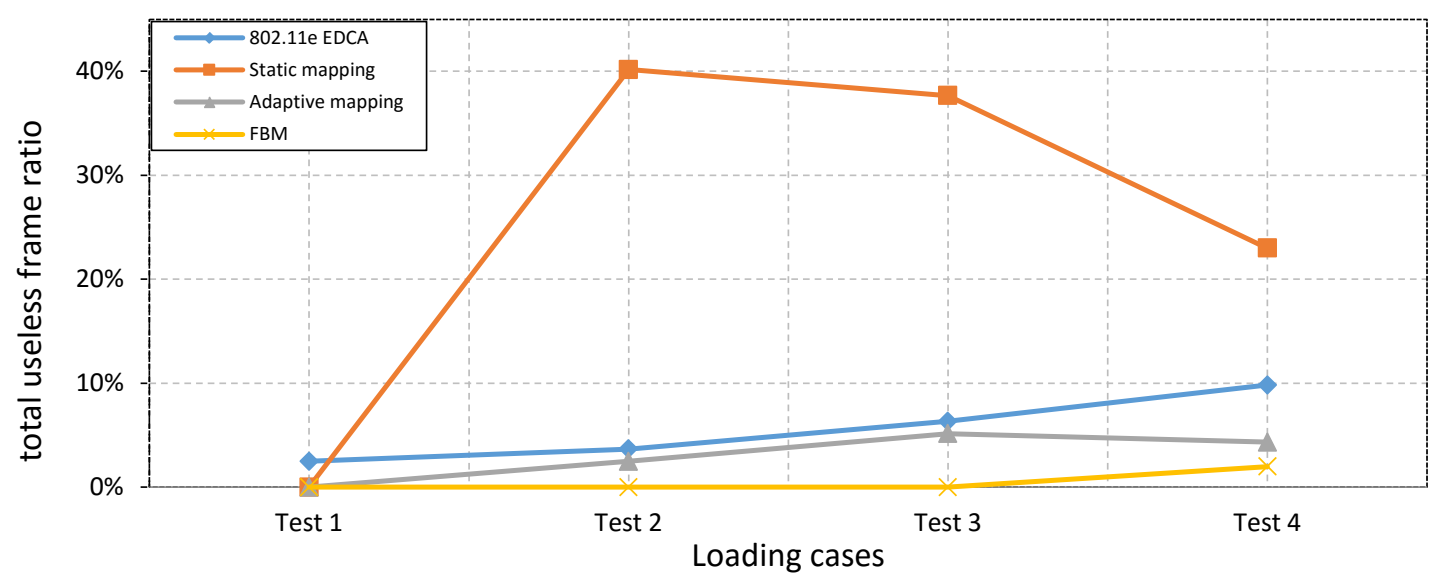

Figure 4. The average useless frame of video sequences under various loading cases (Foreman)

Table 6 presents the average PSNR of the fast-motion video, Football. Table 7 shows the frames and loss of packets of the Football video in test 4 of scenario.

Table 6. The average PSNR in the scenario (Football)

\begin{tabular}{|l|c|c|c|c|}
\hline \multirow{2}{*}{ Loads case } & \multicolumn{4}{|c|}{ Average PSNR (db) } \\
\cline { 2 - 5 } & $\mathbf{8 0 2 . 1 1 e ~ E D C A ~}$ & Static mapping & Adaptive mapping & FBM \\
\hline \hline Test 1 & 23.53 & 28.09 & 23.15 & 28.85 \\
\hline Test 2 & 23.78 & 20.69 & 22.15 & 27.72 \\
\hline Test 3 & 22.88 & 20.09 & 20.38 & 26.72 \\
\hline Test 4 & 22.33 & 20.12 & 20.04 & 25.70 \\
\hline
\end{tabular}

Table 7. The average PSNR and number of frame and packets lost in scenario (Football)

\begin{tabular}{|l|c|c|c|c|c|c|c|c|c|}
\hline \multirow{2}{*}{ mechanism } & \multirow{2}{*}{$\begin{array}{l}\text { Average } \\
\end{array}$} & \multicolumn{4}{|c|}{ Frame loss number } & \multicolumn{4}{|c|}{ Packets loss number } \\
\cline { 3 - 10 } & PSNR (db) & $\boldsymbol{I}$ & $\boldsymbol{P}$ & $\boldsymbol{B}$ & Total & $\boldsymbol{I}$ & $\boldsymbol{P}$ & $\boldsymbol{B}$ & Total \\
\hline \hline 802.11e EDCA & 22.03 & 18 & 29 & 94 & 141 & 103 & 130 & 303 & 536 \\
\hline Static mapping & 20.07 & 0 & 54 & 129 & 182 & 0 & 330 & 481 & 811 \\
\hline Adaptive mapping & 19.62 & 10 & 45 & 159 & 213 & 34 & 109 & 396 & 538 \\
\hline FBM & 25.36 & 1 & 3 & 131 & 135 & 8 & 22 & 534 & 563 \\
\hline
\end{tabular}




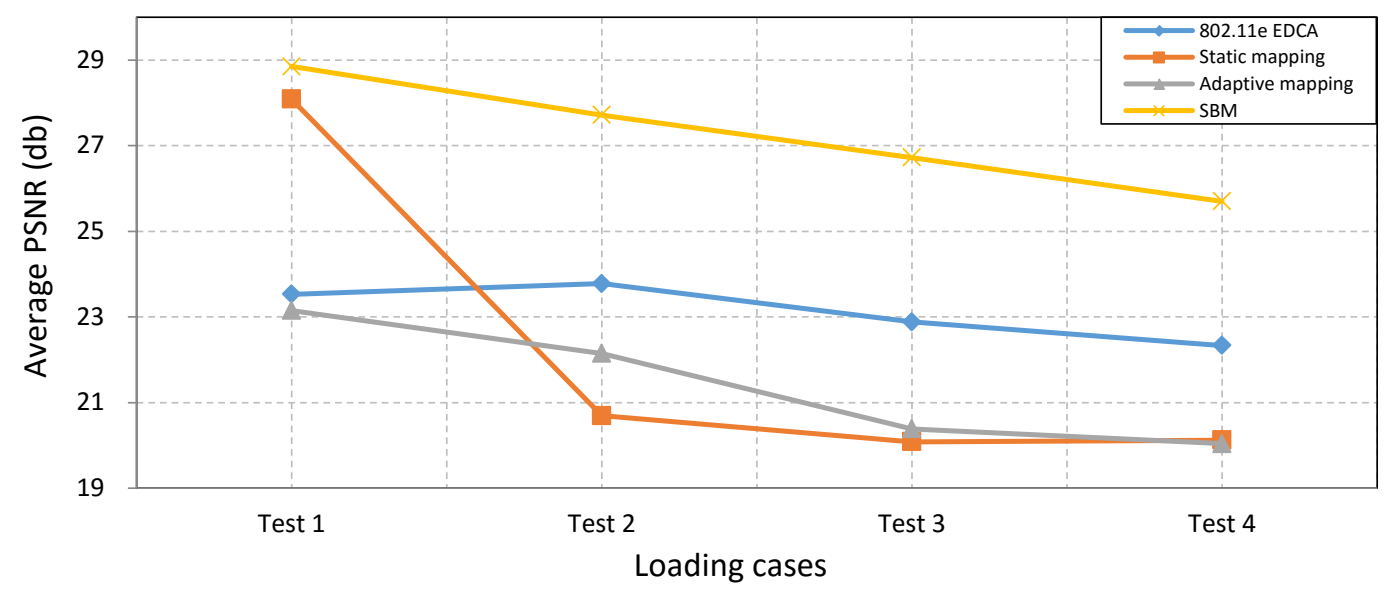

Figure 5. The average PSNR in scenario (Football)

Figure 6 presents the average useless frame of the video sequence Football under various loads. Under heavy network loads with the transmission of fast-motion video sequences, the FBM still yields the lowest proportion of useless frames in the receiver.

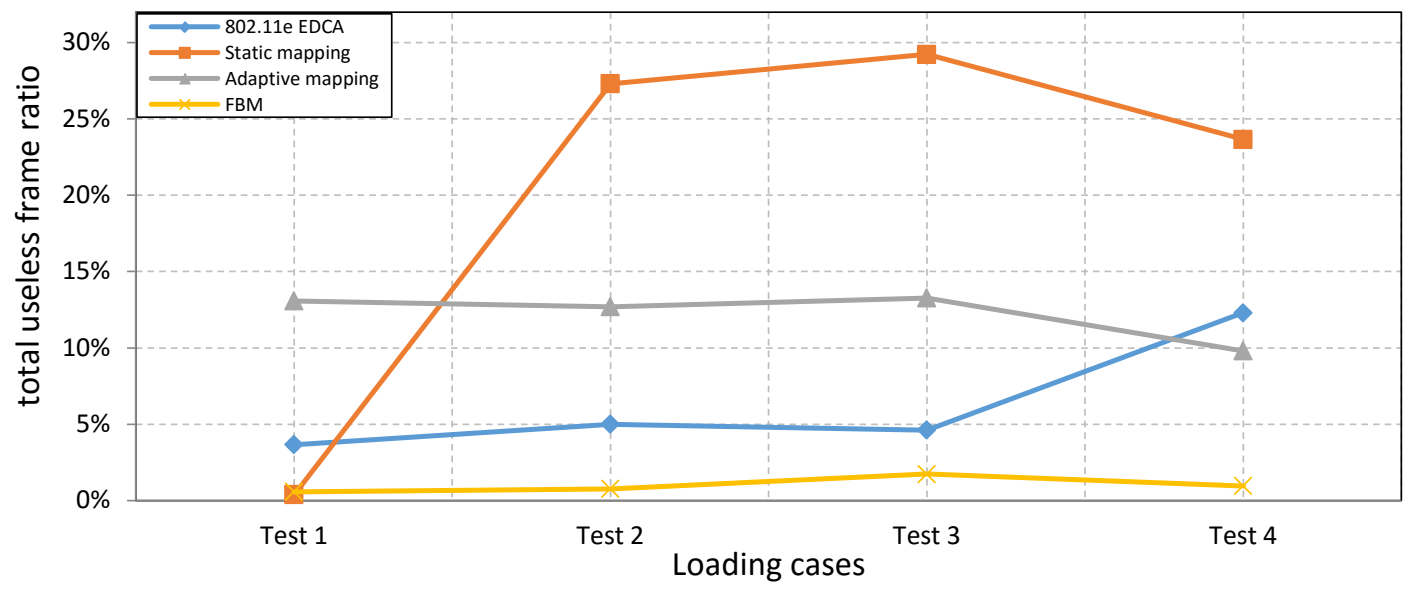

Figure 6. The average useless frame of video sequences under various loading cases (Football)

Figure 7 and Figure 8 present the average power consumed by the transmission of the video sequences under various loading cases. The overflow of the AC_BE and AC_BK queues causes the dropping of the $P$ and $B$ frame packets, as shown in Tables 5 and 8; these packets need not be sent. Hence, static mapping consumes the least power. FBM yields an average PSNR that ranges from $15 \%$ to $25 \%$ better than that of other mechanisms, without depleting more energy in the transmission of the video packets, because the FBM maps the packets of a video frame to the most appropriate AC; transmits the useful packets of the frame; drops the useless packets in the transceiver node to reduce the power consumption associated with transmission, and reserve resources for the useful packets of the video frame. 


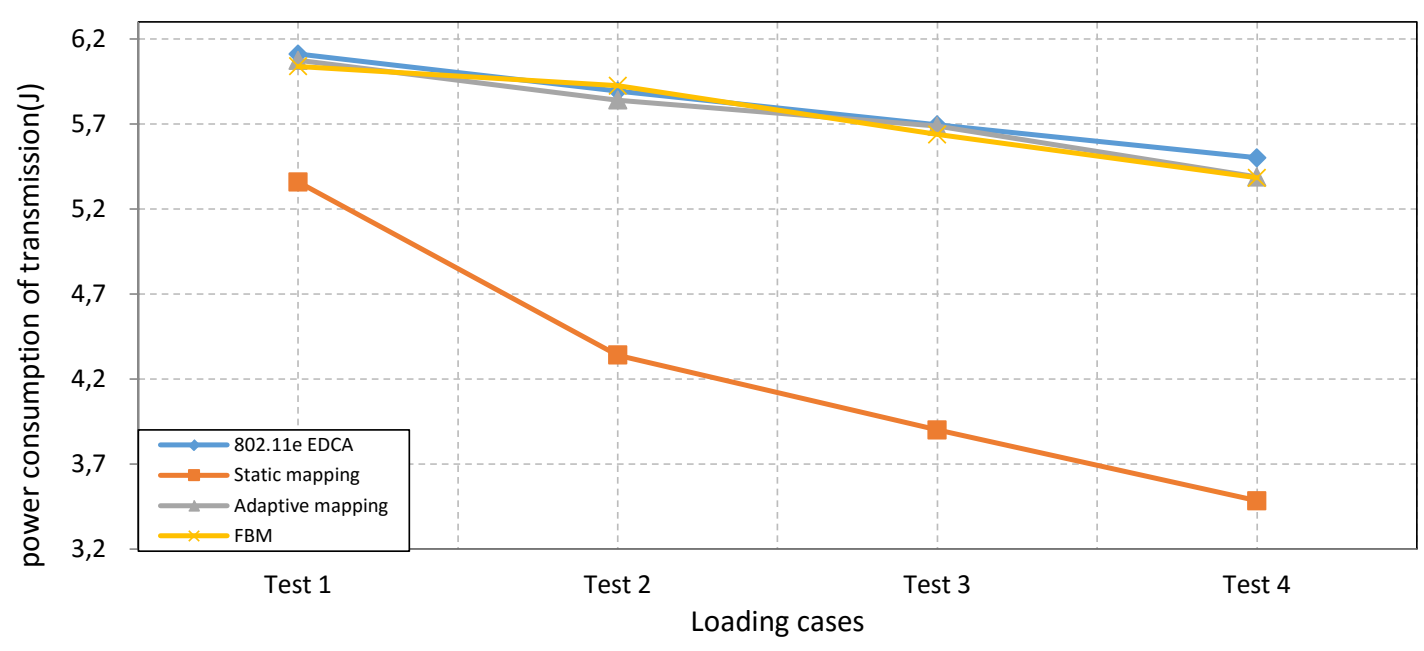

Figure 7. The average power consumption of the transmission of video sequences under various loading cases (Foreman)

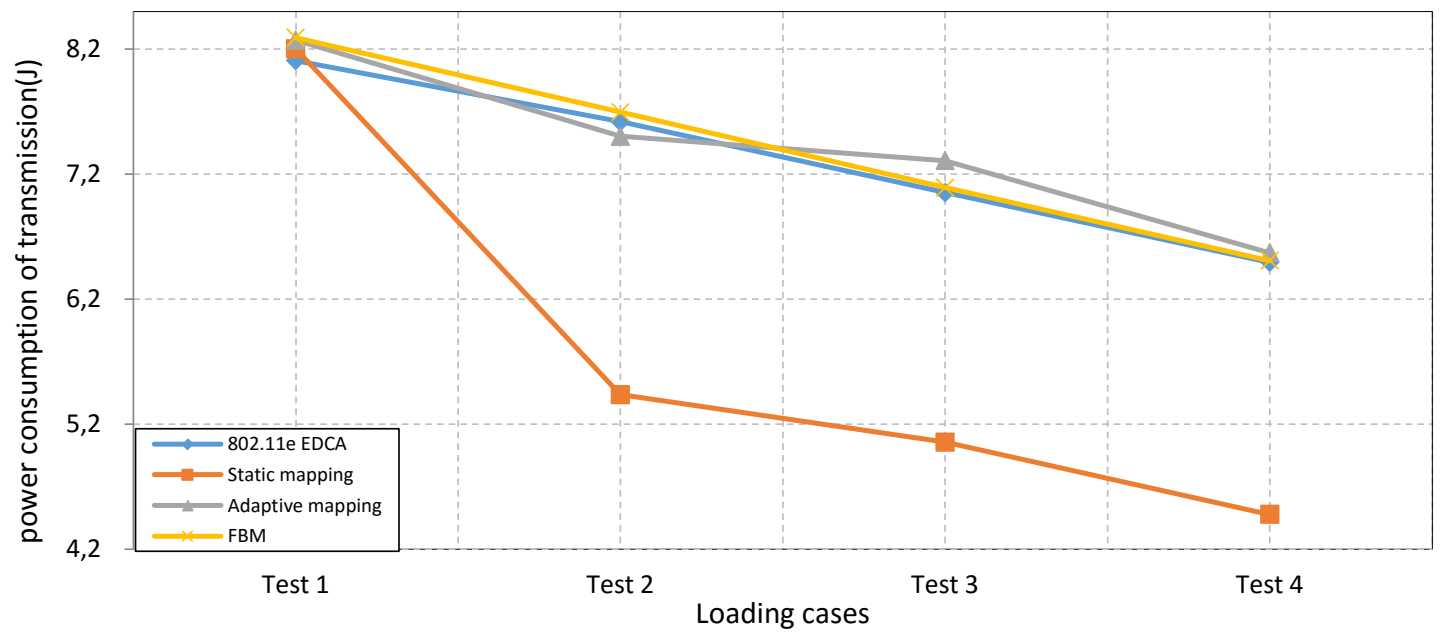

Figure 8. The average power consumption of the transmission of video sequences under various loading cases (Football)

The energy efficiency is analyzed by comparing the relationships between efficiency and traffic load among various mechanisms. Figure 9 and Figure 10 demonstrate that the efficiency of the proposed FBM always exceeds those of other mechanisms, suggesting that the energy of a device that uses this mechanism may be depleted by the transmission of the useful video data (frames that are decodable when received) in WLANs. 


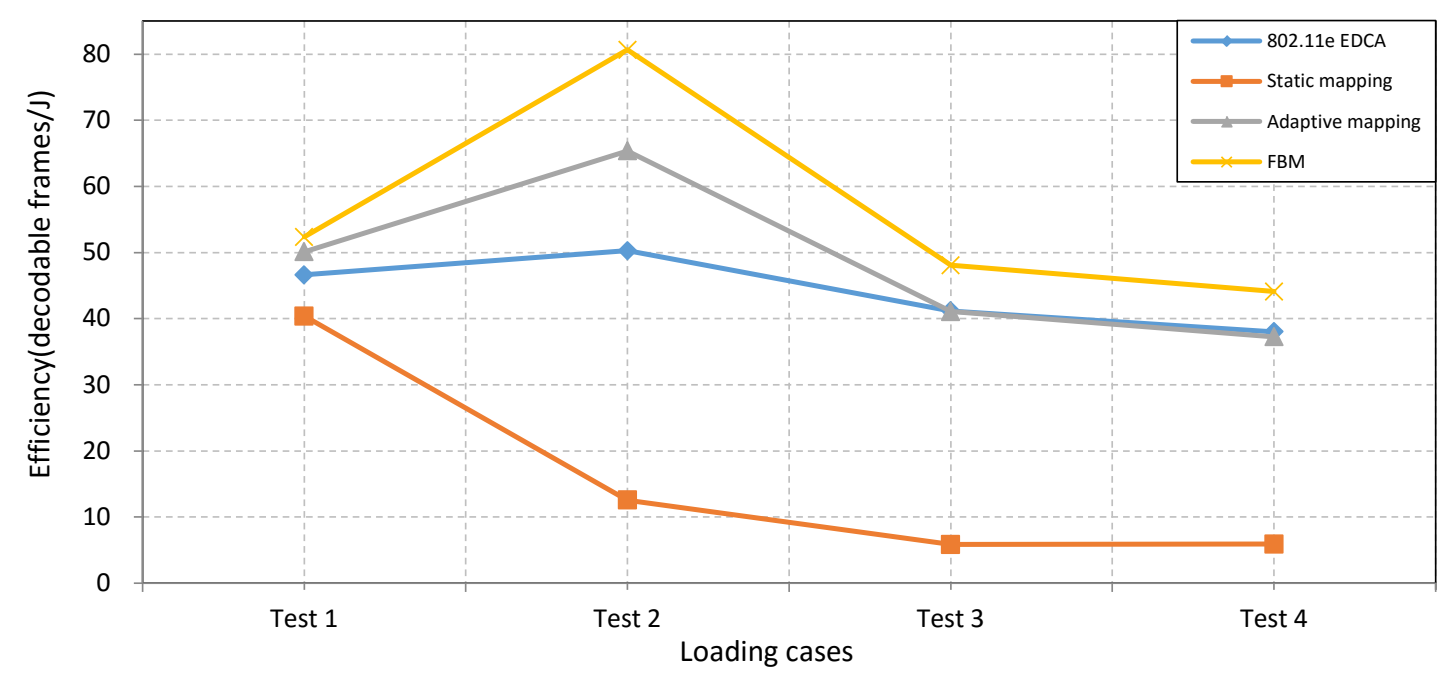

Figure 9. Energy efficiency under various loading cases (Foreman)

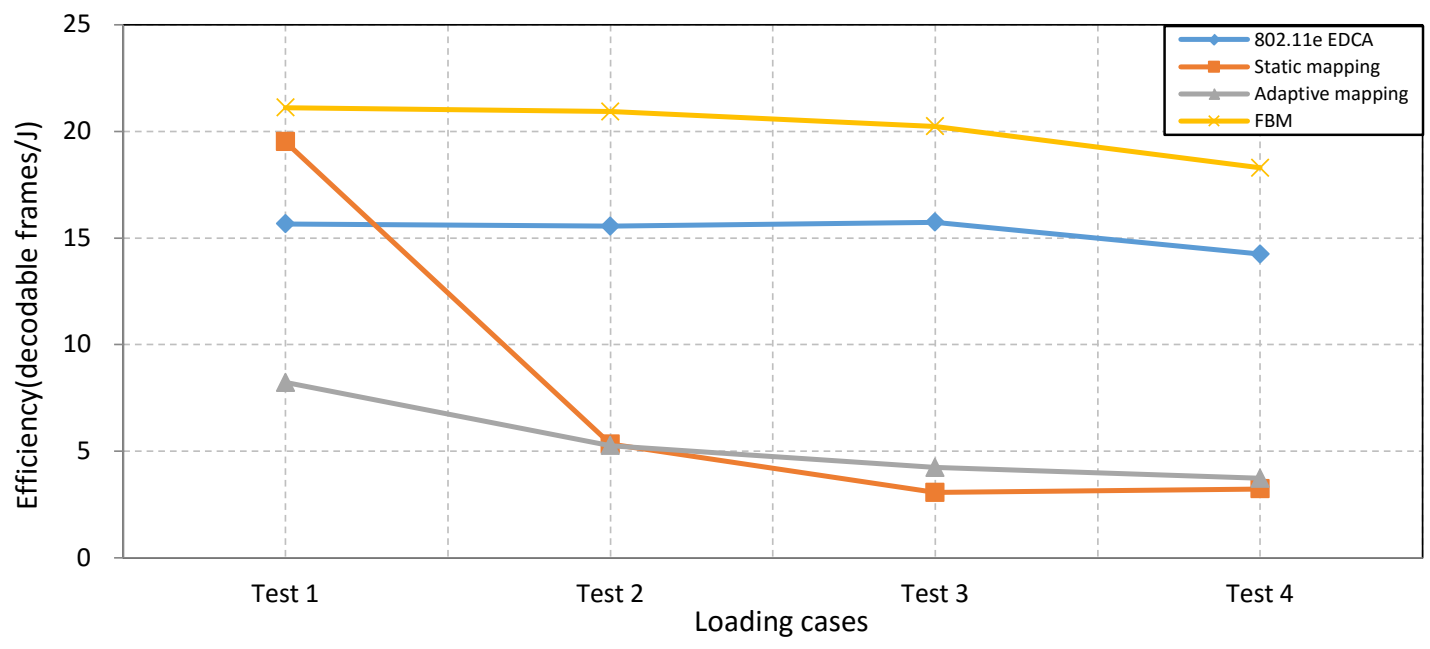

Figure 10 Energy efficiency under various loading cases (Football)

\section{CONCLUSIONS AND FUTURE WORK}

A frame-based mapping mechanism is developed to improve the quality of the MPEG-4 video that is streamed over IEEE 802.11e wireless networks. FBM dynamically allocates video packets to the most appropriate ACs based on the network traffic load, frame type and stream information. Simulations verify that the FBM successfully improves the quality of the transmitted video. The simulation results demonstrate that, under heavy network loading, the FBM scheme outperforms the 802.11e EDCA, static mapping scheme and adaptive mapping scheme in terms of the quality of the transmitted MPEG-4 video.

The results obtained herein concerning the proportion of useless frames received reveal the importance of efficient transmission in 802.11e networks. The proportion of useless frames that are received when the FBM is used is smaller than that obtained using any other mechanism in various scenarios. The simulation results demonstrate that transmission by the FBM is more efficient than by other mechanisms, suggesting 
that the energy of a device in which the mechanism is used may not be wasted in transmitting useless video data in WLANs.

In the future, the authors will further develop more efficient methods for transmitting MPEG traffic in WLANs that consider the dependency of the video frame within a GOP. Our purpose is to apply this research in order to monitor forests and rural areas [31][32].

\section{Acknowledgements:}

Foundation item: The National Project of Taiwan (No.: MOST 103-2221-E507-001). Authors are grateful to Ministry of Science and Technology, Government of Taiwan for financial support to carry out this work.

\section{REFERENCES}

[1] Jaime Lloret, Pedro V. Mauri, Miguel Garcia y Antonio J. Ferrer, Designing WLANS for Video Transmission in Rural Environments for Agriculture and Environmental Researches and Educational Purposes, WSEAS Transactions on Communications, Volume 5, Issue 11, Pp. 2064-2070. November 2006

[2] 170- Marcelo Atenas, Sandra Sendra, Miguel Garcia, Jaime Lloret, IPTV Performance in IEEE 802.11n WLANs, IEEE Global Communications Conference (IEEE Globecom 2010), Miami (USA), 6 - 10 December 2010

[3] Alejandro Canovas, Miguel Garcia, Jaime Lloret, Marcelo Atenas and Rafael Rizo, Improving IPTV QoE taking the suitable MPEG-2/MPEG-4 Quantizer based on Jitter, Delay and lost packets measurements, International Journal On Advances in Telecommunications, Vol. 3, Issue 3\&4, Pp. 129 - 139. December 2010.

[4] Marcelo Atenas, Miguel Garcia, Alejandro Canovas, Jaime Lloret, A MPEG-2/MPEG-4 Quantizer to Improve the Video Quality in IPTV services, The Sixth International Conference on Networking and Services (ICNS 2010), Cancun (Mexico), 7-13 marzo 2010

[5] Alberto Álvarez, Laura Pozueco, Sergio Cabrero, Xabiel García Pañeda, Roberto García, David Melendi, Gabriel Díaz Orueta, Adaptive Streaming: A subjective catalog to assess the performance of objective QoE metrics, Network Protocols and Algorithms, Vol 6, No 2 (2014), Pp. 123-136

[6] Alfio Lombardo, Carla Panarello, Giovanni Schembra, EE-ARQ: a Green ARQ-Based Algorithm for the Transmission of Video Streams on Noise Wireless Channels, Network Protocols and Algorithms, Vol 5, No 1 (2013), Pp. 41-70

[7] Takeuchi, S., Sezaki, K., \& Yasuda, Y. (2005). Dynamic adaptation of contention window sizes in IEEE 802.11e wireless LAN. In Fifth international conference on information, communications and signal processing (ICICSP 2005) (pp. 659-663).

[8] Wang, Y., \& Huang, B. (2004). Performance improvement of IEEE 802.11e. In The 13th IEEE workshop on local and metropolitan area networks, LANMAN 2004 (pp. 51-54), 25-28 April 2004.

[9] Majkowski, J., \& Palacio, F. C. (2006). Enhanced TXOP scheme for efficiency improvement of WLAN IEEE 802.11e. In Vehicular technology conference, VTC-2006 Fall. 2006 IEEE 64th (pp. 1-5), Sept. 2006.

[10] Majkowski, J., \& Palacio, F. C. (2006). Dynamic TXOP configuration for Qos enhancement in IEEE 802.11e wireless LAN. In International conference on software in telecommunications and computer networks, SoftCOM 2006 (pp. 66-70), Sept. 29 2006- Oct. 12006.

[11] Kim, J. O., Tode, H., \& Murakami, K. (2005). Service-based rate adaptation architecture for IEEE 802.11e QoS networks. In IEEE global telecommunications conference (GLOBECOM'05), St. Louis, Missouri, 28 Nov.-2 Dec. 2005. pp. 3341-3345

[12] Siris, V. A., \& Courcoubetis, C. (2006). Resource control for the EDCA mechanism in multi-rate IEEE 802.11e networks. In International symposium on a world of wireless, mobile and multimedia networks, WoWMoM 2006 (pp. 419-428), 26-29 June 2006.

[13] A. KSENTINI, M. NAIMI, A. GUEROUI. Toward an improvement of H.264 video transmission over IEEE 802.11e through a cross-layer architecture. IEEE Communications Magazine, 2006, 44 (1): 107-114.

[14] C. H. Foh, Y. Zhang, Z. Ni, J. Cai, K. N. Ngan. Optimized cross-layer design for scalable video transmission over the IEEE 802.11e networks. IEEE Transactions on Circuits and Systems for Video Technology, 2007, 17 (12): 1665-1678.

[15] H.-L. CHEN, P.-C. LEE, S.-H. HU. Improving scalable video transmission over IEEE 802.11e through a cross-layer architecture, The 4th International Conference on Wireless and Mobile Communications (ICWMC 2008). Athens, Greece, 2008. 
[16] Rakesh Radhakrishnan, Balaji Tirouvengadam, Amiya Nayak, “Cross Layer Design for Efficient Video Streaming over LTE Using Scalable Video Coding”, Network Protocol, and Algorithms, vol.4 , No.4, 2012.

[17] Ghafoor, K. Z., Bakar, K. A., Zainuddin, Z. M., Ke, C. H., \& Gonzalez, A. J. (2012). Reliable Video Geocasting over Vehicular Ad Hoc Networks. Ad Hoc \& Sensor Wireless Networks, 15(2-4), 201-221.

[18] CH Lin, CK Shieh, C.-H. Ke, NK Chilamkurti, S Zeadally, An adaptive cross-layer mapping algorithm for MPEG-4 video transmission over IEEE 802.11e WLAN. Springer Telecommun Syst. 42(2), 353-362 (2009)

[19] Chih-Heng Ke, Chong-Yi Yang, Jiann-Liang Chen. A Stream-Based Mapping Mechanism for MPEG-4 Video Transmission over IEEE 802.11e. In 2014 International Symposium on Computer, Consumer and Control (IS3C), 2014 , 512-515.

[20] N Chilamkurti, S Zeadally, R Soni, G Giambene. Wireless multimedia delivery over 802.11e with cross-layer optimization techniques. Multimedia Tools Appl. 47(1), 189-205 (2010). doi:10.1007/s11042-009-0413-6

[21] Hsing-Lung Chen, Po-Ching Lee, Shu-Hua Hu, "Improving Scalable Video Transmission over IEEE 802.11e through a Cross-Layer Architecture," icwmc, pp.241-246, ICWMC08, 2008.

[22]

[23] Xiao Xiao, Yanlin Xu. A dynamic queue assignment algorithm under IEEE 802.11e. The 2013 2nd International Symposium on Instrumentation and Measurement, Sensor Network and Automation (IMSNA), 2013, 186-189.

[24] Yu Peng, Lianghui Ding, Liang Qian, Feng Yang. Load Adaptive Dynamic Mapping for video streaming over IEEE 802.11e network. The 2013 International Conference on Wireless Communications \& Signal Processing (WCSP), 2013, 1-6

[25] Wen-Ping Lai, En-Cheng Liou, A novel cross-layer design using comb-shaped quadratic packet mapping for video delivery over 802.11e wireless ad hoc networks, EURASIP Journal on Wireless Communications and Networking, 2012

[26] Sunghee Lee, Kwangsue Chung, The Study of Dynamic Video Frame Mapping Scheme for Multimedia Streaming over IEEE 802.11e WLAN. International Journal of Multimedia and Ubiquitous Engineering(Vol. 8, No. 1), January, 2013

[27] X. Yao, W. Wang, S. Yang, Y. Cen, X. Yao, T. Pan, IPB-frame Adaptive Mapping Mechanism for Video Transmission over IEEE 802.11e WLANs. ACM SIGCOMM Computer Communication Review, Volume 44, Number 2, April 2014

[28] A. Ziviani, B. E. Wolfinger, J. F. Rezende, O. C. M. B. Duarte, and S. Fdida, Joint Adoption of QoS Schemes for MPEG Streams, Kluwer Academic Publishers, vol. 26, no. 1, pp. 59-80, May 2005

[29] Mangold, S., Choi, S., Hiertz, G. R., Klein, O., \& Walke, B. (2003). Analysis of IEEE 802.11e for QoS support in wireless LANs. IEEE wireless communications magazine, Dec. 2003. pp. 40-50

[30] The Network Simulator, NS2.29. Available at: http://www.isi.edu/nsnam/ns/

[31] YUV test sequences. Available at: http://videocoders.com/yuv.html

[32] Jaime Lloret, Ignacio Bosch, Sandra Sendra and Arturo Serrano, A Wireless Sensor Network for Vineyard Monitoring That Uses Image Processing, Sensors, Vol. 11, Issue 6, Pp. 6165-6196. June 2011. doi:10.3390/s110606165

[33] Jaime Lloret, Miguel Garcia, Diana Bri and Sandra Sendra, A Wireless Sensor Network Deployment for Rural and Forest Fire Detection and Verification, Sensors, Vol. 9, Issue 11, Pp. 8722-8747. October 2009, doi:10.3390/s91108722 